\title{
Classification of self-dual torsion-free LCA groups
}

\author{
by
}

\author{
Sheng L. W u (Eugene, Oreg.)
}

\begin{abstract}
In this paper we seek to describe the structure of self-dual torsion-free LCA groups. We first present a proof of the structure theorem of self-dual torsion-free metric LCA groups. Then we generalize the structure theorem to a larger class of selfdual torsion-free LCA groups. We also give a characterization of torsion-free divisible LCA groups. Consequently, a complete classification of self-dual divisible LCA groups is obtained; and any self-dual torsion-free LCA group can be regarded as an open subgroup of a well-understood torsion-free divisible LCA group.
\end{abstract}

Introduction. After M. Rajagopalan and T. Soundararajan proved the structure theorem of self-dual torsion-free metric LCA groups in 1969, Corwin (1970) initiated a new and interesting approach to the problem of classifying the self-dual LCA groups in [2]. Though some sufficient and necessary conditions for an extension group $G$ of a compact abelian group $N$ by $\widehat{N}$ to be self-dual were given, the detailed structure of the group remains a mystery. In the last twenty years since the appearance of these two papers, no new progress appears to have been made. The problem of classifying self-dual LCA groups is still sitting in the dark, waiting for some light to be shed on it. We prove a structure theorem for self-dual torsion-free weak $p$-local LCA groups and present a complete classification of self-dual divisible LCA groups.

The paper consists of six sections. Section 1 contains notations and basic definitions used in the paper. In Section 2, we give a brief discussion of direct product and prove a sufficient and necessary condition for a totally disconnected compact abelian group to be decomposed into a direct product of a family of its closed subgroups; this will be needed in Section 5 to characterize the local direct product $\sum_{i \in S}\left(\Omega_{p}^{i}: \Delta_{p}^{i}\right)$ of copies of the group $\Omega_{p}$ of $p$-adic numbers. Section 3 gives a characterization of the group $\Omega_{p}$ of $p$-adic numbers and shows that the direct product $\prod_{i \in S} \Delta_{p}^{i}$ (or $\Delta_{p}^{\mu}$ in short, where $\mu=|S|)$ of copies of the group $\Delta_{p}$ of $p$-adic integers is uniquely determined by the underlying index set and the prime number $p$. Section 4 presents a 
different proof of the structure theorem of self-dual torsion-free metric LCA groups that was first proved by M. Rajagopalan and T. Soundararajan in [5]. Since one can show generally that any self-dual torsion-free LCA group is of the form $R^{n} \times D \times \widehat{D} \times \sum_{i \in S}\left(H_{p}: K_{p}\right)$ with each $H_{p}$ self-dual containing $K_{p}$ as an open and compact subgroup and $D$ being a divisible discrete abelian group, the study of self-dual torsion-free LCA groups boils down to the study of self-dual torsion-free topological $p$-groups $H_{p}$. It is tempting to conjecture that $H_{p}$ is of the form $\sum_{i \in S}\left(\Omega_{p}^{i}: \Delta_{p}^{i}\right)$ since $H_{p}$ contains an open and compact subgroup of the form $\Delta_{p}^{\mu}$ (see $\left.[1,2.8]\right)$. In 1968, Neil Rickert gave an example in [6] (see also Example 2) showing that self-duality and torsion-freeness are not enough for a torsion-free topological $p$-group to be decomposed into a local direct product $\sum_{i \in S}\left(\Omega_{p}^{i}: \Delta_{p}^{i}\right)$ of copies of the group $\Omega_{p}$ of $p$-adic numbers. Hence two questions are raised:

(1) When can a self-dual torsion-free topological $p$-group be decomposed into a local direct product of copies of the group of $p$-adic numbers ?

(2) What other conditions does one need to impose on $G$ in order for $G$ to have a nice structure?

The first question leads us to the discussion of $p$-local and weak $p$-local LCA groups in Section 5. With the success in proving a necessary and sufficient condition for an LCA group to be topologically isomorphic to a local direct product of copies of the group $\Omega_{p}$ of $p$-adic numbers, we generalize M. Rajagopalan and T. Soundararajan's result to a larger class (see Theorem 5.14); whereas, the second question motivates the research in Section 6 of the structure of self-dual divisible LCA groups which turn out to behave as desired (see Corollary 6.14).

Acknowledgements. I am extremely indebted to my advisor, Prof. Kenneth A. Ross, for his invaluable suggestions, help and encouragement. I would like to thank Prof. M. Rajagopalan for providing helpful information with regard to my research.

1. Notations and definitions. All groups used in the paper are assumed to be locally compact abelian (abbreviated as LCA) groups unless otherwise indicated. Most of the notations and concepts in the paper can be found in [1] or [3]. We shall repeat some for the sake of clarity. $T$ denotes the unit circle group with Euclidean topology. $R^{n}$ is the usual Euclidean group $(n \geq 1)$. Let $p$ be a prime number; then $Z\left(p^{n}\right)$ represents the cyclic group of order $p^{n}, Z\left(p^{\infty}\right)$ is the quasicyclic group of the rationals expressible in the form $m / p^{n}$ under addition $\bmod 1$. We shall adopt [3]'s notation $\Omega_{p}$ and $\Delta_{p}$ (or $\Lambda_{0}$ ) for the group of $p$-adic numbers and the group of $p$-adic integers, respectively. When there is a need to use $\Lambda_{k}$, which is the subgroup of $\Omega_{p}$ that consists of all $x=\left(x_{n}\right) \in \Omega_{p}$ such that $x_{n}=0$ for all integers 
$n<k$ (see [3] for details), we shall use $\Lambda_{0}$ instead of $\Delta_{p}$ together with $\Lambda_{k}$. The character group of an LCA group $G$ with the compact-open topology is denoted by $\widehat{G}$. If $H$ is a subgroup of $G, H^{\perp}$ (or $A(\widehat{G}, H)$, to avoid confusion) denotes the annihilator of $H$ in $\widehat{G}$. Let $A$ be a subset of an LCA group $G$; then $\langle A\rangle$ denotes the subgroup of $G$ generated by $A$, while $\langle A\rangle^{-}$represents the closed subgroup of $G$ generated by $A$. The identity component of an LCA group $G$ is denoted by $C(G)$.

Let $\left\{G_{i}\right\}_{i \in S}$ be a family of LCA groups; then $\prod_{i \in S} G_{i}$ indicates the direct product of all $G_{i}$ 's with the product topology. For simplicity, especially when there is no need to use the underlying indices $i$, we shall write $G^{\mu}$ for $\prod_{i \in S} G_{i}$ if $G_{i}=G$ for all $i \in S$, where $\mu=|S|$ is the cardinality of the index set $S$. If $\left\{G_{i}\right\}_{i \in S}$ is a family of discrete abelian groups, then the weak direct sum of all $G_{i}$ 's is denoted by $\sum_{i \in S} G_{i}$. But when $G_{i}=G$ for all $i \in S$ and there is no need for using underlying indices, we shall write $G^{\mu *}$ instead of $\sum_{i \in S} G_{i}$ for simplicity.

Definition 1.1. Let $G$ be an LCA group. By the $p$-component $G_{p}$ of $G$ we mean the set of all $x \in G$ such that $\lim _{n \rightarrow \infty} p^{n} x=0$. $G$ is said to be a topological p-group if and only if $G_{p}=G$ for some fixed prime number $p$.

Definition 1.2. Let $G$ be an LCA group and $G !=\left\{x \in G: \lim _{n \rightarrow \infty} n ! x\right.$ $=0\}$. Then $G$ is said to be a topological torsion group if and only if $G !=G$.

DEFINITION 1.3. A topological isomorphism, denoted by $\simeq$, is both a group isomorphism and a homeomorphism of topological spaces. Two LCA groups are said to be topologically isomorphic if there exists a topological isomorphism between them.

DeFinition 1.4. Let $G$ be an LCA group and let $\widehat{G}$ be its character group. If $G$ and $\widehat{G}$ are topologically isomorphic, then $G$ is said to be selfdual.

Definition 1.5. Let $\left\{G_{i}\right\}_{i \in S}$ and $\left\{H_{i}\right\}_{i \in S}$ be two families of LCA groups with each $H_{i}$ open in $G_{i}$ and let $G=\left\{\left(x_{i}\right) \in \prod_{i \in S} G_{i}: x_{i} \in H_{i}\right.$ for all but a finite number of indices $i\}$. Topologize $G$ so that $\prod_{i \in S} H_{i}$ is an open subgroup of $G$. Then $G$ is called the local direct product of the $G_{i}$ 's with respect to the open subgroups $H_{i}$ 's, denoted by $\sum_{i \in S}\left(G_{i}: H_{i}\right)$.

2. Direct product. One important way of understanding topological groups is to decompose them into the Cartesian product of simpler ones whenever possible. Our main concern in this section is to try to find some necessary and sufficient conditions for a compact topological group to be decomposed into a direct product of a family of its closed normal subgroups.

The topological groups mentioned in this section need not be abelian unless it is indicated explicitly. 
Definition 2.1. Let $G$ be a topological group and let $\left\{G_{i}: i \in S\right\}$ be a family of normal closed subgroups of $G$ such that $G=\left\langle\left\{G_{i}: i \in S\right\}\right\rangle^{-}$. If $|S| \geq \aleph_{0}$, then we also assume $G$ is compact. If there exists a topological isomorphism $\tau: G \rightarrow \prod_{i \in S} G_{i}$ such that for all $j \in S$ and $x \in G_{j}, \tau(x)=$ $\left(y_{i}\right)$, where $y_{i}=x$ when $i=j$ and $y_{i}=0$ when $i \neq j$, then we will say that $G$ can be decomposed into the direct product of its closed subgroups $\left\{G_{i}: i \in S\right\}$ and write $G=\bigoplus_{i \in S} G_{i}$.

Suppose that $G$ is the Cartesian product of a family of compact groups $\left\{K_{i}: i \in S\right\}$, i.e., $G=\prod_{i \in S} K_{i}$. Let $G_{i}=K_{i} \times \prod_{j \neq i}\{0\}$; then by definition $G=\bigoplus_{i \in S} G_{i}$.

Definition 2.2. Let $G$ be a locally compact topological group, let $\left\{M_{i}\right.$ : $i \in S\}$ be a family of closed normal subgroups of $G$ and $Q_{i}=\left\langle\left\{M_{j}: j \in S\right.\right.$, $j \neq i\}\rangle^{-} . \quad\left\{M_{i}: i \in S\right\}$ are said to be topologically independent (TI) if $M_{i} \cap Q_{i}=\{0\}$ for each $i \in S$; and they are strongly topologically independent (STI) if $\bigcap_{i \in S} Q_{i}=\{0\}$.

EXAMPLE 1. Let $G=G_{1} \times \ldots \times G_{n}$ and $M_{i}=\{0\} \times \ldots \times\{0\} \times G_{i} \times$ $\{0\} \times \ldots \times\{0\}$; then $\left\{M_{i}: i=1, \ldots, n\right\}$ are both TI and STI.

By definition we can see easily that $\left\{M_{i}: i \in S\right\}$ being STI implies $\left\{M_{i}: i \in S\right\}$ being TI, but not the other way around. Since the example we have in hand to explain this is not trivial, we will wait until after proving Theorem 5.13. But it is not suprising that, in the case when the index set $S$ is finite and the underlying group $G$ is compact, TI and STI are equivalent. We will demonstrate this in the following proposition. Proposition 2.3 and Theorem 2.4 are known, but somewhat obscure in the literature and so we include brief proofs.

Proposition 2.3. Let $\left\{M_{i}: i \in S\right\}$ be a finite set of closed normal subgroups of a compact group $G$ (not necessarily abelian); then $\left\{M_{i}: i \in S\right\}$ are $T I$ if and only if $\left\{M_{i}: i \in S\right\}$ are STI.

Proof. Without loss of generality we may assume that $G=\left\langle\left\{M_{i}\right.\right.$ : $i \in S\}\rangle$; otherwise we can consider the closed subgroup $G_{1}=\left\langle\left\{M_{i}\right.\right.$ : $i \in S\}\rangle$. We need only show that TI implies STI. Actually, by [3, 6.12] we know that there exists a topological ispmorphism $\tau: G \rightarrow \prod_{i=1}^{n} M_{i}$ such that $\tau(x)=(0, \ldots, 0, x, 0, \ldots, 0)$, where $x \in M_{i}$. But this implies that $\tau\left(Q_{i}\right)=M_{1} \times \ldots \times M_{i-1} \times\{0\} \times M_{i+1} \times \ldots \times M_{n}$. So $\tau\left(\bigcap_{i=1}^{n} Q_{i}\right)=$ $\bigcap_{i=1}^{n} \tau\left(Q_{i}\right)=\{0\}$. Hence $\bigcap_{i=1}^{n} Q_{i}=\{0\}$ since $\tau$ is an isomorphism. So $\left\{M_{i}: i \in S\right\}$ are STI.

It turns out that strong topological independence is a reasonable condition imposed on a family of compact subgroups in order for the group to be decomposed into a direct product of this family of compact subgroups. Here 
is a theorem concerning direct decomposition proved by Pontryagin (see $[4, \S 21 . \mathrm{E}]$

THEOREM 2.4. Let $G$ be a compact topological group and let $\left\{M_{i}: i \in S\right\}$ be a family of closed normal subgroups of $G$; then $G=\bigoplus_{i \in S} M_{i} \Leftrightarrow G=$ $\left\langle\left\{M_{i}: i \in S\right\}\right\rangle^{-}$and $\left\{M_{i}: i \in S\right\}$ are $S T I$.

Proof. The sufficiency is the result of $[4, \S 21 . E]$. Now let us prove the necessity. Assume $G=\bigoplus_{i \in S} M_{i}$. Then by our Definition 2.1 we know that $G=\left\langle\left\{M_{i}: i \in S\right\}\right\rangle^{-}$and there exists a natural topological isomorphism $\tau$ from $G$ onto $\prod_{i \in S} M_{i}$. Let $Q_{i}=\left\langle\left\{M_{j}: j \neq i\right\}\right\rangle^{-}$; then it is clear that $\tau\left(Q_{i}\right)=\{0\} \times \prod_{j \neq i} M_{j}$. Therefore $\tau\left(\bigcap_{i \in S} Q_{i}\right)=\bigcap_{i \in S} \tau\left(Q_{i}\right)=\{0\}$. Hence $\bigcap_{i \in S} Q_{i}=\{0\}$, and the necessity is proved.

For a totally disconnected LCA group $G$, the following theorem gives a satisfactory answer to the question "Under what conditions does TI imply STI?". It also gives necessary and sufficient conditions for a compact totally disconnected LCA group to be decomposed into a direct product of a family of its closed subgroups. It will play an important role in our characterization of self-dual torsion-free LCA groups.

THEOREM 2.5. Let $G$ be a compact totally disconnected LCA group and let $\left\{M_{i}: i \in S\right\}$ be a family of closed subgroups of $G$ such that $G=\left\langle\left\{M_{i}\right.\right.$ : $i \in S\}\rangle^{-}$. Then the following are equivalent:

(a) (1) for any open neighborhood $U$ of 0 in $G$ all $M_{i}$ 's are contained in $U$ except for finitely many indices;

(2) $\left\{M_{i}: i \in S\right\}$ are $T I$;

(b) $\left\{M_{i}: i \in S\right\}$ are $S T I$ (hence $G=\bigoplus_{i \in S} M_{i}$ ).

Proof. $(\mathrm{b}) \Rightarrow(\mathrm{a})$ is clear. For $(\mathrm{a}) \Rightarrow(\mathrm{b})$, we need to show that $\bigcap_{i \in S} Q_{i}=$ $\{0\}$ by Definition 2.2, where $Q_{i}=\left\langle\left\{M_{j}: j \neq i\right\}\right\rangle^{-}$. Let $g \in \bigcap_{i \in S} Q_{i}$; then for any open neighborhood $V$ of 0 , there exists an open and compact subgroup $U$ contained in $V$ by $[3,7.7]$ since $G$ is totally disconnected. For this compact open subgroup $U$ almost all $M_{i}$ 's are contained in it by assumption (1). Assume that $M_{i_{k}} \nsubseteq U$ for $k=1, \ldots, m$ and $M_{i} \subseteq U$ for all $i \notin\left\{i_{1}, \ldots, i_{m}\right\}$. We claim that $g \in Q_{i_{1} \ldots i_{m}}=\left\langle\left\{M_{i}: i \notin\left\{i_{1}, \ldots, i_{m}\right\}\right\}\right\rangle^{-}$. Indeed, starting from $Q_{i_{1}}$ we know that $g \in Q_{i_{1}}$ since $g \in \bigcap_{i \in S} Q_{i}$. Also it is clear that $Q_{i_{1}}=M_{i_{2}}+Q_{i_{1} i_{2}}$. So there exist $x_{2} \in M_{i_{2}}$ and $y_{2} \in Q_{i_{1} i_{2}}$ such that $g=x_{2}+y_{2}$. Note that $y_{2} \in Q_{i_{1} i_{2}} \subseteq Q_{i_{2}}$ and $g \in Q_{i_{2}}$, so we must have $x_{2}=g-y_{2} \in M_{i_{2}} \cap Q_{i_{2}}=\{0\}$ by assumption (2). So $x_{2}=0$ and $g=y_{2} \in Q_{i_{1} i_{2}}$. Proceeding in this way $m-1$ times we will have $g \in Q_{i_{1} \ldots i_{m}}$. Since $U$ contains all $M_{i}$ 's with $i \notin\left\{i_{1}, \ldots, i_{m}\right\}$ and $U$ is an open and compact (hence closed) subgroup of $G$ we must have $Q_{i_{1} \ldots i_{m}} \subseteq U$, which implies that $g \in U \subseteq V$. Since $V$ is arbitrary, we must have $g=0$. 
So $\bigcap_{i \in S} Q_{i}=\{0\}$, i.e., $\left\{M_{i}: i \in S\right\}$ are STI. Now by Theorem 2.4 we know that $G=\bigoplus_{i \in S} M_{i}$.

\section{Some characterizations of $\Omega_{p}$ and topological $p$-groups}

Lemma 3.1. Let $G$ be a torsion-free topological p-group. Suppose that there exists a sequence $\left\{u_{n}\right\}_{n=0}^{\infty}$ so that $u_{0} \neq 0, p u_{n}=u_{n-1}$ for $n \geq 1$ and $p^{n} u_{0} \rightarrow 0(n \rightarrow \infty)$; then $H=\left\langle\left\{u_{n}: n \geq 0\right\}\right\rangle^{-}$is topologically isomorphic to $\Omega_{p}$.

Proof. Since $p^{n} u_{0} \rightarrow 0(n \rightarrow \infty)$ and $G$ is totally disconnected (see $[1,2.7])$ there exists an open compact subgroup $K \ni u_{0} \notin K$ and $p^{m} u_{0} \in K$ for some $m \geq 1$.

Let $N$ be the smallest positive integer such that $p^{N} u_{0} \in K$ and $p^{N-1} u_{0} \notin K$.

Claim 1. $K \cap H=\left\langle p^{N} u_{0}\right\rangle^{-}$.

Proof. First of all $\left\langle p^{N} u_{0}\right\rangle^{-} \subseteq K \cap H$. Now if there exists $x \notin\left\langle p^{N} u_{0}\right\rangle^{-}$ such that $x \in K \cap H$, then $\exists y \in\left\langle\left\{u_{n}: n \geq 0\right\}\right\rangle=H_{1}$ such that $y \in K \cap H$ and $y \notin\left\langle p^{N} u_{0}\right\rangle^{-}$(because $K \cap H$ is an open neighborhood of $x,\left\langle p^{N} u_{0}\right\rangle^{-}$ is closed in $K \cap H$ and $H_{1}$ is dense in $H$ ).

For convenience let $v_{0}=p^{N} u_{0}, v_{1}=p^{N-1} u_{0}, \ldots, v_{N}=u_{0}, v_{N+1}=$ $u_{1}, \ldots$, and write

$$
y=l_{1} v_{1}+l_{2} v_{2}+\ldots+l_{n} v_{n}+t
$$

where $t \in\left\langle p^{N} u_{0}\right\rangle^{-}, 0<\left|l_{n}\right|<p$ and $1 \leq n \leq N$ (this is possible since $y \notin\left\langle p^{N} u_{0}\right\rangle^{-}$). Without loss of generality we may assume $l_{n}>0$ (otherwise we can consider $-y)$. Note that

$$
\begin{aligned}
p^{n-1} y & =p^{n-1}\left(l_{1} v_{1}+\ldots+l_{n-1} v_{n-1}+t\right)+l_{n} p^{n-1} v_{n} \\
& =l_{n} v_{1}+z \in K \cap H,
\end{aligned}
$$

where $z=p^{n-1}\left(l_{1} v_{1}+\ldots+l_{n-1} v_{n-1}+t\right) \in\left\langle p^{N} u_{0}\right\rangle^{-} \subseteq K \cap H$. Therefore $l_{n} v_{1}=p^{n-1} y-z \in K \cap H$, which implies that $v_{1} \in K \cap \bar{H}$, i.e., $p^{N-1} u_{0} \in K$, a contradiction. Hence Claim 1 is proved.

Claim 2. $\left\langle\left\{v_{1}, v_{2}, \ldots\right\} \cup\left\langle v_{0}\right\rangle^{-}\right\rangle \simeq \Omega_{p}$.

Proof. First of all, Claim 1 shows that the closed subgroup $\left\langle v_{0}\right\rangle^{-}$is a compact open subgroup of $H$. By [1, Lemma 2.11], $\left\langle v_{0}\right\rangle^{-} \simeq \Delta_{p}$. Let $\varphi$ be the topological isomorphism. Now define

$$
\psi:\left\langle\left\{v_{1}, v_{2}, \ldots\right\} \cup\left\langle v_{0}\right\rangle^{-}\right\rangle \rightarrow \Omega_{p}
$$

by

$$
l_{n} v_{n}+l_{n-1} v_{n-1}+\ldots+l_{1} v_{1}+h \mapsto y+\varphi(h),
$$


where $0<l_{n}<p, 0 \leq l_{i}<p(i=1, \ldots, n-1)$ and

$$
y=\left(\ldots, 0, l_{n}, l_{n-1}, \ldots, l_{1}, 0, \ldots\right) .
$$

It is straightforward to show that $\varphi$ is well defined and is an algebraic isomorphism. Note that $\left\langle\left\{v_{1}, v_{2}, \ldots\right\} \cup\left\langle v_{0}\right\rangle^{-}\right\rangle$is open, hence closed in $H$, so we must have $H=\left\langle\left\{v_{1}, v_{2}, \ldots\right\} \cup\left\langle v_{0}\right\rangle^{-}\right\rangle$. So $H \simeq \Omega_{p}$.

The following lemma will be used a lot in our study of self-dual torsionfree topological $p$-groups. For simplification, we shall write $\Delta_{p}^{i}$ (or $\Delta_{p}^{j}$ ) for $\Delta_{p}$, where $i$ (or $j$ ) is an index, while $\Delta_{p}^{\mu}$ (or $\Delta_{p}^{m}$ ) is the direct product of $\mu$ (or $m$ ) copies of $\Delta_{p}$ as defined in Section 1 .

LEMma 3.2. Two compact topological p-groups $\prod_{i \in S} \Delta_{p}^{i}$ and $\prod_{i \in A} \Delta_{p}^{i}$ are topologically isomorphic if and only if $|S|=|A|$.

Proof. We need only show that the condition is necessary. Assume that $\prod_{i \in S} \Delta_{p}^{i} \simeq \prod_{i \in A} \Delta_{p}^{i}$; then by taking duals we have $\sum_{i \in S} Z\left(p^{\infty}\right) \simeq$ $\sum_{i \in A} Z\left(p^{\infty}\right)$. Now by infinite abelian group theory we have $|S|=|A|$.

LEMMA 3.3. Let $H$ be any open compact subgroup of $G=\sum_{i \in S}\left(\Omega_{p}^{i}: \Delta_{p}^{i}\right)$. Then $H \simeq \prod_{i \in S} \Delta_{p}^{i}$.

Proof. Let $H$ be any open and compact subgroup of $G$. Note that $G$ is the local direct product of copies of the group of $p$-adic numbers. By the way the topology of $G$ is defined $G$ contains $\prod_{i \in S} \Delta_{p}^{i}$ as a compact and open subgroup. So by $[1,2.23], H \simeq \prod_{i \in S} \Delta_{p}^{i}$.

Proposition 3.4. Let $G$ be a torsion-free topological p-group and let $K$ be an open and compact subgroup that is topologically isomorphic to $\prod_{i \in S} \Delta_{p}^{i}$, where $\Delta_{p}^{i}=\Delta_{p} \forall i \in S$, with $|S| \leq \aleph_{0}$ and $G / K \simeq \sum_{i \in S} Z\left(p^{\infty}\right)$. Then $G$ satisfies the second axiom of countability.

Proof. First of all we know that $K$ is second countable since by [3, Theorem 24.14], $w(K)=w(\widehat{K}) \leq \aleph_{0}$. It is clear that $G=\bigcup_{i \in S}\left(x_{i}+K\right)$, where $x_{i}$ 's are fixed representatives of all those countably many cosets. Now by $[3,8.19]$ the proposition is proved.

4. Self-dual torsion-free metric LCA groups. The following lemma is actually a special case of a lemma proved by Neil W. Rickert in [6]. For convenience we will state it here for later use.

LEMMA 4.1. Let $G$ be a torsion-free $L C A$ topological p-group and let $K$ be a compact subgroup. Assume that a subgroup $H$ of $G / K$ is isomorphic to $Z\left(p^{\infty}\right)$. Then there is a closed subgroup $H^{\prime}$, isomorphic to $\Omega_{p}$, which maps onto the group $H$ under the natural map of $G$ onto $G / K$. 
Pro of. Note that any torsion-free topological $p$-group is a $\Delta_{p}$-module; the lemma follows from [6, Lemma 4].

LEMMA 4.2. Let $G$ be a metric torsion-free topological p-group that is selfdual; then $G$ is topologically isomorphic to a local direct product of countably many copies of the group of p-adic numbers.

Proof. Let $K$ be a fixed open and compact subgroup of $G$. Then by [1, Proposition 2.8], $K \simeq \prod_{i \in S} \Delta_{p}^{i}$. Since $G$ is metrizable we must have $|S| \leq \aleph_{0}$. Since $G$ is self-dual it follows, by $[1,2.23]$, that $(G / K)^{\wedge} \simeq K^{\perp} \simeq$ $K \simeq \prod_{i \in S} \Delta_{p}^{i}$ and so $G / K \simeq \sum_{i \in S} Z^{i}\left(p^{\infty}\right)$, where each $Z^{i}\left(p^{\infty}\right)=Z\left(p^{\infty}\right)$. So $G$ satisfies the second axiom of countability by Proposition 3.4. Now by Lemma 4.1 for each $i \in S$ there is a closed subgroup $L_{i}$ of $G$ such that $L_{i} \simeq \Omega_{p}$ and $\left(L_{i}+K\right) / K \simeq Z^{i}\left(p^{\infty}\right)$. Clearly $G=\left\langle\left\{L_{i}: i \in S\right\} \cup K\right\rangle$. Next we need to show that

$$
G=\left\{\bigcap_{n=1}^{\infty} p^{n} G\right\}^{-}=\bigcap_{n=1}^{\infty} \overline{p^{n} G}
$$

so that we can draw our conclusion by [8, Part I, Theorem 3]. For this purpose let $L=\left\langle L_{i}: i \in S\right\rangle$.

Claim. $\bar{L}=G$.

Proof. If $G \neq \bar{L}$, then $G / \bar{L}=(\bar{L}+K) / \bar{L} \neq\{0\}$ and so $G / \bar{L}$ is the image of the compact group $K$. Hence $G / \bar{L}$ is compact. The fact that $G$ is a topological $p$-group implies that $G$ is totally disconnected by $[1,2.7]$. So by $[3,3.5]$ we know that $G$ is 0 -dimensional, which implies that $G / \bar{L}$ is 0 dimensional by $[3,7.11]$. Therefore it is totally disconnected by [1, P.27(d)]. So by $[3,24.26],(G / \bar{L})^{\wedge}=A(\widehat{G}, \bar{L})$ is a torsion group, which implies that $\widehat{G}$ contains elements of finite order, a contradiction. Therefore $G=\bar{L}$.

Now by the fact that the $L_{i}$ 's are divisible we know that for any $n$, $p^{n} G \supseteq p^{n} L=L$. Hence

$$
\bigcap_{n=1}^{\infty} p^{n} G \supseteq L, \quad \overline{p^{n} G} \supseteq \bar{L}=G
$$

Thus

$$
\begin{gathered}
G \supseteq\left\{\bigcap_{n=1}^{\infty} p^{n} G\right\}^{-} \supseteq \bar{L}=G, \\
G \supseteq \bigcap_{n=1}^{\infty} \overline{p^{n} G} \supseteq \bar{L}=G,
\end{gathered}
$$


which implies that

$$
G=\left\{\bigcap_{n=1}^{\infty} p^{n} G\right\}^{-}=\bigcap_{n=1}^{\infty} \overline{p^{n} G} .
$$

Now by $[8$, Part I, Theorem 3]

$$
(G: K) \simeq \sum_{i \in S}\left(\Omega_{p}^{i}: \Delta_{p}^{i}\right),
$$

where $|S| \leq \aleph_{0}$.

THEOREM 4.3. Let $G$ be a torsion-free metric LCA group. Then $G$ is self-dual if and only if

$$
G \simeq R^{n} \times D \times \widehat{D} \times \sum_{p \in \wp}\left(\sum_{i \in K_{p}}\left(\Omega_{p}^{i}: \Delta_{p}^{i}\right): \Delta_{p}^{\mu_{p}}\right),
$$

where $\wp$ is a subset of primes and for each $p \in \wp, K_{p}$ is an index set with cardinal $\mu_{p} \leq \aleph_{0} ; \Omega_{p}^{i}=\Omega_{p}$ and $\Delta_{p}^{i}=\Delta_{p} \forall i \in K_{p}$ and $p \in \wp ; D$ is a torsion-free divisible countable discrete group.

Proof. $\Leftarrow$ is obvious. For $\Rightarrow$, by [4, Lemmas 10 and 11],

$$
G \simeq R^{n} \times D \times \widehat{D} \times \sum_{p \in \wp}\left(G_{p}: H_{p}\right),
$$

where $D$ is a torsion-free countable divisible discrete group (since $G$ is metrizable) and $\wp$ is a subset of prime numbers; $G_{p}$ is a self-dual topological $p$-group with open and compact subgroup $H_{p} \forall p \in \wp$. Now by Lemma 4.2 there exists an index set $K_{p}$ with $\left|K_{p}\right| \leq \aleph_{0}$ such that

$$
\left(G_{p}: H_{p}\right) \simeq \sum_{i \in K_{p}}\left(\Omega_{p}^{i}: \Delta_{p}^{i}\right)
$$

Note. The above theorem was first proved by M. Rajagopalan and T. Soundararajan in [5] where a different approach was used.

5. Self-dual torsion-free $p$-local and weak $p$-local LCA groups. In this section, we study $p$-local and weak $p$-local groups. It turns out that any self-dual torsion-free countable $p$-local group is a local direct product of countably many groups of $p$-adic numbers. Hence the class of torsionfree self-dual countable $p$-local LCA groups and the class of self-dual metric torsion-free topological $p$-groups coincide. We shall give a characterization of the local direct product of copies of the group $\Omega_{p}$ of $p$-adic numbers (see Theorem 5.13), then generalize M. Rajagopalan and T. Soundararajan's result to a somewhat larger class without much effort (see Theorem 5.14).

It is well known that any torsion-free topological $p$-group $G$ contains an open compact subgroup $K$ that is topologically isomorphic to $\prod_{i \in S} \Delta_{p}^{i}$ 
for some index set $S$. So algebraically $G$ can be regarded as a subgroup of $\prod_{i \in S} \Omega_{p}^{i}$ (see Proposition 5.6 below) with $\prod_{i \in S} \Delta_{p}^{i}$ as an open compact subgroup. Since for any index set $S$ the local direct product $\sum_{i \in S}\left(\Omega_{p}^{i}: \Delta_{p}^{i}\right)$ is self-dual, it is natural to ask whether $G$ must be a local direct product of groups of $p$-adic numbers if $G$ is a self-dual torsion-free topological $p$-group. Unfortunately, the answer is negative. Here is an example given by Neil Rickert in [6] that shows that being a torsion-free self-dual topological $p$ group is not enough for an LCA group to be decomposed into a local direct product of groups of $p$-adic numbers.

EXAmple 2. Let $G=\left\{x \in \prod_{i=1}^{\infty} \Omega_{p}^{i}: \exists m \in N\right.$ such that $p^{m} x \in$ $\left.\prod_{i=1}^{\infty} \Delta_{p}^{i}\right\}$, where $\Omega_{p}^{i}=\Omega_{p}$ and $\Delta_{p}^{i}=\Delta_{p} \forall i \geq 1$. Topologize $G$ so that $\prod_{i=1}^{\infty} \Delta_{p}^{i}$ is an open compact subgroup of $G$. Then $G \times \widehat{G}$ is a torsion-free self-dual topological $p$-group, but it is not topologically isomorphic to a local direct product of groups of $p$-adic numbers (for details see [6]).

The group defined in Example 2 is exactly a special case of the groups $B_{p}(\mu)$ with $\mu=\aleph_{0}$ that will be studied in Section 6 , which play an important role in the characterization of divisible torsion-free LCA groups and self-dual divisible LCA groups.

Definition 5.1. Let $G$ be an LCA group; $G$ is called a $p$-local group if $G$ contains an open subgroup that is topologically isomorphic to a local direct product $\sum_{i \in S}\left(\Omega_{p}^{i}: \Delta_{p}^{i}\right)$. If $|S| \leq \aleph_{0}$, then $G$ is said to be countably p-local.

The following proposition is an immediate result of the definition.

Proposition 5.2. If $G$ is a local direct product of groups of $p$-adic numbers, i.e., $G=\sum_{i \in S}\left(\Omega_{p}^{i}: \Delta_{p}^{i}\right)$, where $S$ is an index set with $\Omega_{p}^{i}=\Omega_{p}$ and $\Delta_{p}^{i}=\Delta_{p}$ for all $i \in S$, then $G$ is $p$-local.

Definition 5.3. Let $G$ be an LCA group. $G$ is said to be weak p-local if any $p$-component of $G / C(G)$ is zero or countably $p$-local.

Before proving the main theorem for weak $p$-local groups, we shall give some sufficient conditions for an LCA group to be $p$-local and show that if a countably $p$-local group is self-dual then it is actually topologically isomorphic to a local direct product of countably many copies of the group of $p$-adic numbers.

Proposition 5.4. If $G$ is a divisible torsion-free topological p-group, then $G$ is p-local.

P r o of. Let $H$ be any open compact subgroup of $G$. Then by [1, Proposition 2.8] $H$ is topologically isomorphic to $\prod_{i \in S} \Delta_{p}^{i}$ for some index set $S$. 
Let $\tau$ be the topological isomorphism. Since $G$ is divisible, $\tau$ can be extended to an algebraic homomorphism $\varphi$ of $\sum_{i \in S}\left(\Omega_{p}^{i}: \Delta_{p}^{i}\right)$ into $G$ by $[3$, A.7]. Indeed, one can show easily that $\varphi$ is a topological monomorphism of $\sum_{i \in S}\left(\Omega_{p}^{i}: \Delta_{p}^{i}\right)$ into $G$. So $G$ is $p$-local.

Proposition 5.4 will be obvious when we are able to describe the structure of divisible torsion-free topological $p$-groups in Theorem 6.2.

Proposition 5.5. Let $G$ be an LCA group, and let $\left\{M_{i}\right\}_{i \in S}$ and $\left\{L_{i}\right\}_{i \in S}$ be two families of closed subgroups of $G$ such that each $M_{i}$ is a proper open and compact subgroup of $L_{i} \quad \forall i \in S$. If $\left\{L_{i}\right\}_{i \in S}$ are topologically independent and $\left\langle\left\{M_{i}\right\}_{i \in S}\right\rangle^{-}$is a compact and open subgroup of $G$ such that $\left\langle\left\{M_{i}\right\}_{i \in S}\right\rangle^{-}=\bigoplus_{i \in S} M_{i}$, then the local direct product $\sum_{i \in S}\left(L_{i}: M_{i}\right)$ is topologically isomorphic to an open subgroup of $G$.

Pr o of. For convenience we still denote $L_{i} \times \prod_{j \neq i}\{0\}$ by $L_{i}$ (an obvious abuse of notation) and we also write $\bar{x}_{j}=\left(y_{i}\right)$ with $y_{i}=x_{j}$ when $i=j$ and $y_{i}=0$ when $i \neq j$. First of all, by the definition of local direct product we know that for any $x \in \sum_{i \in S}\left(L_{i}: M_{i}\right), x$ can be written uniquely as $x=\bar{x}_{i_{1}}+\ldots+\bar{x}_{i_{n}}+y$, where $\bar{x}_{i_{k}} \in L_{i_{k}} \backslash M_{i_{k}}(k=1, \ldots, n)$ and $y \in$ $\{0\} \times \ldots \times\{0\} \times \prod_{j \notin\left\{i_{1}, \ldots, i_{n}\right\}} M_{j}$. Define

$$
\varphi: \sum_{i \in S}\left(L_{i}: M_{i}\right) \rightarrow G
$$

by

$$
\varphi(x)=x_{i_{1}}+\ldots+x_{i_{n}}+\tau(y) .
$$

Then $\varphi$ is clearly well defined by the above discussion. It is straightforward to show that $\varphi$ is a homomorphism and one-to-one. Since the restriction of $\varphi$ to $\prod_{i \in S} M_{i}$ is a topological monomorphism and $\prod_{i \in S} M_{i}$ is an open compact subgroup of $\sum_{i \in S}\left(L_{i}: M_{i}\right), \varphi$ is actually a topological monomorphism. Hence the proposition is proved.

Proposition 5.6. Any torsion-free topological p-group $G$ can be regarded as an algebraic subgroup of $\prod_{i \in S} \Omega_{p}^{i}$ for some index set $S$ with $\prod_{i \in S} \Delta_{p}^{i}$ topologically isomorphic to an open and compact subgroup of $G$.

Proof. Let $K$ be an open and compact subgroup of $G$. Then by [1, Proposition 2.8], $K$ is topologically isomorphic to $\prod_{i \in S} \Delta_{p}^{i}$ for some index set $S$. Let $\tau$ be the topological isomorphism of $K$ onto $\prod_{i \in S} \Delta_{p}^{i}$. Since $\prod_{i \in S} \Delta_{p}^{i}$ is an algebraic subgroup of the divisible group $\prod_{i \in S} \Omega_{p}^{i}, \tau$ can be extended to a group homomorphism $\varphi$ of $G$ into $\prod_{i \in S} \Omega_{p}^{i}$ by $[3$, A.7]. It is easy to show that $\varphi$ is one-to-one. Hence $\varphi$ is a monomorphism of $G$ into $\prod_{i \in S} \Omega_{p}^{i}$ 
Proposition 5.7. Let $G$ be a torsion-free $L C A$ group and let $p$ be a fixed prime. Let $G$ contain an open subgroup $H$ which is a local direct product $\sum_{i \in S}\left(J_{p}^{i}: K_{p}^{i}\right)$ of a family $\left\{J_{p}^{i}\right\}_{i \in S}$ of LCA groups with each $K_{p}^{i}$ open and compact in $J_{p}^{i}$. For each $i \in S$ let $J_{p}^{i} \simeq \Omega_{p}$. Suppose further that $G$ contains a family $\left\{D_{\alpha}\right\}_{\alpha \in A}$ such that the following hold:

(1) $|A| \leq|S| \leq \aleph_{0}$;

(2) $D_{\alpha} \simeq \Omega_{p}$ for every $\alpha \in A$;

(3) the algebraic subgroup generated by the set $H \cup\left(\bigcup_{\alpha \in A} D_{\alpha}\right)$ is $G$.

Then $G$ is topologically isomorphic to the local direct product $\sum_{i \in S}\left(\Omega_{p}^{i}: \Delta_{p}^{i}\right)$ of the group of p-adic numbers.

Proof. Let $K=\prod_{i \in S} K_{p}^{i}$. Then $K$ is compact and open in $H$ and hence is open in $G$. We claim that $G / K \simeq \sum_{i \in S} Z^{i}\left(p^{\infty}\right)$.

Indeed, $G / K=H / K+(D+K) / K$, where $D=\left\langle\left\{D_{\alpha}: \alpha \in A\right\}\right\rangle$. It is clear that $H / K$ is divisible. Also one can show that $(D+K) / K$ is divisible. Therefore $G / K$ is a divisible discrete topological $p$-group. Since $H / K \simeq \sum_{i \in S} Z^{i}\left(p^{\infty}\right)$ and $(D+K) / K=\sum_{\alpha \in A}\left(D_{\alpha}+K\right) / K=$ $\sum_{\alpha \in A} Z^{\alpha}\left(p^{\infty}\right)$ by [3, 5.32], where $|A| \leq|S|$ by our assumption (1), we must have $G / K \simeq \sum_{i \in S} Z^{i}\left(p^{\infty}\right)$ by [3, A.14]. But this implies that $G$ satisfies the second axiom of countability by Proposition 3.4. Now let $L=\left\langle\left\{J_{p}^{i}\right\}_{i \in S} \cup\left\{D_{\alpha}\right\}_{\alpha \in A}\right\rangle$; here we still use $J_{p}^{i}$ to denote its corresponding isomorphic image in $\sum_{i \in S}\left(J_{p}^{i}: K_{p}^{i}\right)$. Then $L$ is a dense divisible subgroup of $G$ since it is easy to see that $\left\langle\left\{J_{p}^{i}\right\}_{i \in S}\right\rangle$ is dense in $\sum_{i \in S}\left(J_{p}^{i}: K_{p}^{i}\right)$. So $p^{n} G \supseteq$ $p^{n} L=L$ for any non-negative integer $n$. Therefore $G \supseteq \overline{p^{n} L}=\bar{L}=G$ and $G \supseteq\left\{\bigcap p^{n} G\right\}^{-}=\bar{L}=G$. Consequently, $G=\left\{\bigcap_{n=1}^{\infty} p^{n} G\right\}^{-}=\bigcap_{n=1}^{\infty} \overline{p^{n} G}$. By [8, Part I, Theorem 3] we know that $G$ is topologically isomorphic to a local direct product of countably many copies of the group of $p$-adic numbers.

Remark. Proposition 5.7 is [5, Lemma 8] which should have been stated with the underlying index set countable.

Proposition 5.8. Let $G$ be a torsion-free LCA group that is countably p-local. Then $G$ is self-dual if and only if $G$ is topologically isomorphic to a local direct product of countably many copies of the group of p-adic numbers.

Proof. It is obvious that we need only prove the sufficiency. Let $H$ be the open subgroup that is isomorphic to a local direct product $\sum_{i \in S}\left(\Omega_{p}^{i}\right.$ : $\left.\Delta_{p}^{i}\right)$ of countably many copies of the group of $p$-adic numbers $\left(|S| \leq \aleph_{0}\right)$ and let $K$ be the compact open subgroup of $H$ that is topologically isomorphic to $\prod_{i \in S} \Delta_{p}^{i}$ under the same topological isomorphism. Then $K$ is an open compact subgroup of $G$ since $H$ is open in $G$. Consequently, $G / K$ is discrete. 
Since $(G / K)^{\wedge} \simeq K^{\perp}$ and $G$ is self-dual, $K^{\perp}$ is topologically isomorphic to an open and compact subgroup of $G$, hence is topologically isomorphic to $K$ by $[1,2.23]$. Therefore $(G / K)^{\wedge} \simeq \prod_{i \in S} \Delta_{p}^{i}$. By taking duals we have $G / K \simeq \sum_{i \in S} Z\left(p^{\infty}\right)$. Since $|S| \leq \aleph_{0}, \sum_{i \in S} Z\left(p^{\infty}\right)$ is countable and $K$ is second countable, $G$ must be second countable. Now by the same process as in the proof of Lemma 4.2 we know that $G$ is topologically isomorphic to a local direct product of countably many copies of the group of $p$-adic numbers.

Note that in the proof of the sufficiency we do not assume that $G$ is metric at all. But it turns out that $G$ is automatically a metric group, assuming that $G$ is countably $p$-local torsion-free self-dual.

The condition that $G$ be self-dual cannot be omitted. The group $G$ in Example 2 is not a local direct product of the group of $p$-adic numbers. But it is clearly a countably $p$-local group.

It is very reasonable to conjecture that if a torsion-free topological $p$ group is $p$-local (not necessarily countably $p$-local) and self-dual, then the group must be topologically isomorphic to a local direct product of copies of the group of $p$-adic numbers. So far no proof of the conjecture has been found, nor has any counterexample been given. We strongly believe that the answer to this conjecture would be affirmative if one can confirm that the following conditions (1) and (2) imply (a), assuming that $G$ is a compact torsion-free topological $p$-group and $\left\{M_{i}\right\}_{i \in S}$ is a family of compact subgroups of $G$ such that $G=\left\langle\left\{M_{i}: i \in S\right\}\right\rangle^{-}$. Let $Q_{i}=\left\langle\left\{M_{j}: j \neq i\right\}\right\rangle^{-}$. The above-mentioned conditions are:

(1) $G \simeq \prod_{i \in S} M_{i}$;

(2) $M_{i} \cap Q_{i}=\{0\} \quad \forall i \in S$;

(a) $\bigcap_{i \in S} Q_{i}=\{0\}$.

If it is necessary one can also assume that all $M_{i}$ are topologically isomorphic to $\Delta_{p}$. We do not know whether the answer to the last question is positive or negative. What we have found out is that (2) itself does not imply (a). The counterexample is quite complicated; we will wait until after proving Theorem 5.13.

Here is the structure theorem for torsion-free weak $p$-local self-dual LCA groups.

THEOREM 5.9. Let $G$ be a torsion-free LCA group that is weak p-local. Then $G$ is self-dual if and only if

$$
G \simeq R^{n} \times D \times \widehat{D} \times \sum_{p \in \wp}\left(\sum_{i \in S_{p}}\left(\Omega_{p}^{i}: \Delta_{p}^{i}\right): \Delta_{p}^{\mu_{p}}\right),
$$

where $n$ is a non-negative integer; $D$ is divisible, torsion-free and discrete; $\wp$ is a set of prime numbers; $\Omega_{p}^{i}=\Omega_{p}$ and $\Delta_{p}^{i}=\Delta_{p} \forall i \in S_{p}$, where $S_{p}$ is a 
countable index set with cardinality $\mu_{p}$ depending on $p \in \wp$.

Proof. $\Leftarrow$ is clear. For $\Rightarrow$, by $[5$, Lemmas 10 and 11$]$ we have

$$
G \simeq R^{n} \times D \times \widehat{D} \times \sum_{p \in \wp}\left(H_{p}: K_{p}\right)
$$

where $n$ is a non-negative integer; $D$ is divisible, torsion-free and discrete; $\wp$ is a set of prime numbers for which $H_{p} \neq\{0\}$ and $H_{p}$ is a closed subgroup of $G$ that is torsion-free self-dual with open and compact subgroup $K_{p}$ and is a topological $p$-group in its own right. Since $C(G) \simeq R^{n} \times \widehat{D}$ we have $G / C(G) \simeq D \times \sum_{p \in \wp}\left(H_{p}: K_{p}\right)$. But $D=Q^{m *}$ by [3, A.14], so the topological $p$-subgroup of $G / C(G)$ must be topologically isomorphic to $H_{p}$. Therefore by our assumption that $G$ is weak $p$-local and Proposition 5.8 we have $\left(H_{p}: K_{p}\right) \simeq \sum_{i \in S_{p}}\left(\Omega_{p}^{i}: \Delta_{p}^{i}\right)$ with $K_{p} \simeq \Delta_{p}^{\mu_{p}}$ under the same isomorphism, where $\left|S_{p}\right| \leq \aleph_{0}$. Hence

$$
G \simeq R^{n} \times D \times \widehat{D} \times \sum_{p \in \wp}\left(\sum_{i \in S_{p}}\left(\Omega_{p}^{i}: \Delta_{p}^{i}\right): \Delta_{p}^{\mu_{p}}\right) .
$$

In the rest of this section by using Theorem 2.5 we are able to obtain a sufficient and necessary condition for an LCA topological $p$-group to be decomposed into a local direct product of copies of the group of $p$-adic numbers. To avoid confusion in the following, $A\left(\widehat{G}_{i}, H_{i}\right)$ is used for the annihilator of $H_{i}$ (as a subgroup of $G_{i}$ ) in $\widehat{G}_{i}$ while $H_{i}^{\perp}$ shall be regarded as the annihilator of $H_{i}$ (as a subgroup of $G$ ) in $\widehat{G}$.

Definition 5.10. Let $G$ be a torsion-free topological $p$-group. If $G$ contains an open and compact subgroup $K$ and another open subgroup $H$ containing $K$ such that $(H: K) \simeq \sum_{i \in S}\left(\Omega_{p}^{i}: \Delta_{p}^{i}\right)$, and when $G$ is regarded as an algebraic subgroup of $\prod_{i \in S} \Omega_{p}^{i}$, for any open and compact subgroup $U \subseteq K^{\perp}$

$$
A(G, U) \subseteq\left(\Delta_{p}^{i} \times \prod_{j \neq i} \Omega_{p}^{j}\right) \cap G
$$

for all $i \in S$ except finitely many indices, then $G$ is said to have the finite property.

If $G$ is any torsion-free LCA group and each $p$-component of $G / C(G)$ is either 0 or has the finite property, then $G$ is said to have the finite property.

LEMMA 5.11. Let $H$ be an open and compact subgroup of an LCA group $G$ such that $(G: H)=\sum_{i \in S}\left(G_{i}: H_{i}\right)$ is a local direct product of LCA groups $G_{i}$ 's. Then

(1) $\left(\widehat{G}: H^{\perp}\right)=\sum_{i \in S}\left(\widehat{G}_{i}: A\left(\widehat{G}_{i}, H_{i}\right)\right)$; 
(2) for any open and compact subgroup of $\widehat{G}$ of the form

$$
K_{n}=K_{i_{1}} \times \ldots \times K_{i_{n}} \times \prod_{i \notin\left\{i_{1}, \ldots, i_{n}\right\}} A\left(\widehat{G}_{i}, H_{i}\right),
$$

where each $K_{i_{j}}$ is an open and compact subgroup of $A\left(\widehat{G}_{i_{j}}, H_{i_{j}}\right)$,

$$
A\left(G, K_{n}\right)=A\left(G_{i_{1}}, K_{i_{1}}\right) \times \ldots \times A\left(G_{i_{n}}, K_{i_{n}}\right) \times \prod_{i \notin\left\{i_{1}, \ldots, i_{n}\right\}} H_{i} .
$$

Proof. (1) is a result proved in [3, 23.33]. To show (2), let

$$
E=A\left(G_{i_{1}}, K_{i_{1}}\right) \times \ldots \times A\left(G_{i_{n}}, K_{i_{n}}\right) \times \prod_{i \notin\left\{i_{1}, \ldots, i_{n}\right\}} H_{i} .
$$

It is clear that $E \subseteq A\left(G, K_{n}\right)$. Now for any $x \in G \backslash E$, it suffices to show that $x \notin A\left(G, K_{n}\right)$. For this $x=\left(x_{i}\right)$ there exists $x_{l}$ such that $x_{l} \notin H_{l}$ when $l \notin\left\{i_{1}, \ldots, i_{n}\right\}$, or $x_{l} \notin A\left(G_{i_{j}}, K_{i_{j}}\right)$, when $l=i_{j}$ for some $i_{j} \in\left\{i_{1}, \ldots, i_{n}\right\}$. In the first case, since $x_{l} \notin H_{l}$, there exists a $y_{l} \in A\left(\widehat{G}_{l}, H_{l}\right)$ such that $y_{l}\left(x_{l}\right) \neq 1$. Now let $y=\left(z_{i}\right)$, where $z_{i}=y_{l}$ when $i=l$ and $z_{i}=0$ when $i \neq l$; then $y \in K_{n}$ and $y(x)=y_{l}\left(x_{l}\right) \neq 1$. So $x \notin A\left(G, K_{n}\right)$. In the second case, without loss of generality we may assume that $l=i_{1}$. Since $x_{l} \notin A\left(G_{i_{1}}, K_{i_{1}}\right)$, there exists some $y_{l} \in K_{i_{1}}$ such that $y_{l}\left(x_{l}\right) \neq 1$, and we conclude the proof just as in the first case.

Let $y_{m}=(\ldots, 0,1,0, \ldots)(m=0,1,2, \ldots)$, where the $(1-m)$ th coordinate is 1 . Define a character of $\Omega_{p}$ by $\chi_{m}(x)=\chi_{y_{m}}(x)$ for any $x \in \Omega_{p}$, as in [3, 25.1(9)]. Then each $\chi_{m}$ is a continuous character of $\Omega_{p}$ and for $u_{n}=(\ldots, 0,1,0, \ldots)(n=0,1,2, \ldots)$, where the $(-n)$ th coordinate is 1 , $\chi_{m}\left(u_{n}\right)=e^{2 \pi i / p^{n+m}}$.

Lemma 5.12. (i) For any $y \in p^{m} \Lambda_{0}$, we have $\chi_{m}(y)=1$;

(ii) $E=\left\{\chi_{m}: m=0,1,2, \ldots\right\}$ separates points of $\Omega_{p}$.

Proof. (i) Since $\Lambda_{0}$ is a monothetic group with $u_{0}$ as a generator, the subgroup $\left\langle u_{0}\right\rangle$ generated by $u_{0}$ is dense in $\Lambda_{0}$. Note that $p^{m} \Lambda_{0}$ is a continuous image of the compact LCA group $\Lambda_{0}$, the image $p^{m}\left\langle u_{0}\right\rangle$ of $\left\langle u_{0}\right\rangle$ under the same map must be also dense in $p^{m} \Lambda_{0}$. Now for any $z \in p^{m}\left\langle u_{0}\right\rangle$, $z=p^{m} k u_{0}$ for some integer $k$. So

$$
\chi_{m}(z)=\chi_{m}\left(p^{m} k u_{0}\right)=\left[\chi_{m}\left(u_{0}\right)\right]^{k p^{m}}=\left(\exp \left(2 \pi i / p^{m}\right)\right)^{k p^{m}}=1 .
$$

Therefore $\chi_{m}(y)=1 \forall y \in p^{m} \Lambda_{0}$ since $\chi_{m}$ is continuous on $p^{m} \Lambda_{0}$.

(ii) For any $x \in \Omega_{p}$ with $x \neq 0$, let $x=\left(\ldots, 0, x_{m}, x_{m+1}, \ldots\right)$, where $x_{m} \neq 0$. If $m<0$, then $\chi_{0}(x) \neq 1$. If $m \geq 0$, then $\chi_{m}(x) \neq 1$. Therefore (ii) is proved. 
Here is the theorem characterizing the local direct product of copies of the group of $p$-adic numbers.

THEOREM 5.13. Let $G$ be a torsion-free topological p-group. Then $G$ has the finite property if and only if $G \simeq \sum_{i \in S}\left(\Omega_{p}^{i}: \Delta_{p}^{i}\right)$ and hence is self-dual.

Proof. $\Leftarrow$ Assume that $G \simeq \sum_{i \in S}\left(\Omega_{p}^{i}: \Delta_{p}^{i}\right)$ with respect to $K=$ $\prod_{i \in S} \Lambda_{0}^{i} ;$ then

$$
K^{\perp}=\prod_{i \in S} A\left(\Omega_{p}^{i}, \Lambda_{0}^{i}\right)=\prod_{i \in S} \Lambda_{1}^{i} .
$$

Let $U$ be any open and compact subgroup of $K^{\perp}$; then $U$ contains an open and compact subgroup of the form $\Lambda_{k_{1}}^{i_{1}} \times \ldots \times \Lambda_{k_{n}}^{i_{n}} \times \prod_{i \notin\left\{i_{1}, \ldots, i_{n}\right\}} \Lambda_{1}^{i}$, denoted by $K_{n}$. Then it is clear that $A(G, U) \subseteq A\left(G, K_{n}\right)$. Note that by Lemma 5.11

$$
A\left(G, K_{n}\right)=\Lambda_{-k_{1}+1}^{i_{1}} \times \ldots \times \Lambda_{-k_{n}+1}^{i_{n}} \times \prod_{i \notin\left\{i_{1}, \ldots, i_{n}\right\}} \Lambda_{0}^{i} .
$$

So for each $i \notin\left\{i_{1}, \ldots, i_{n}\right\}$,

$$
A\left(G, K_{n}\right) \subseteq\left(\Lambda_{0}^{i} \times \prod_{j \neq i} \Omega_{p}^{j}\right) \cap G .
$$

Therefore

$$
A(G, U) \subseteq\left(\Lambda_{0}^{i} \times \prod_{j \neq i} \Omega_{p}^{j}\right) \cap G \quad \forall i \notin\left\{i_{1}, \ldots, i_{n}\right\} .
$$

Hence the sufficiency is proved.

$\Rightarrow$ Let $G$ be a torsion-free topological $p$-group that has the finite property; then $G$ can be regarded as an algebraic subgroup of $\prod_{i \in S} \Omega_{p}^{i}$ for some index set $S$ with an open and compact subgroup $K=\prod_{i \in S} \Lambda_{0}^{i}$ contained in an open subgroup $H=\sum_{i \in S}\left(\Omega_{p}^{i}: \Lambda_{0}^{i}\right)$. And for any open and compact subgroup $U \subseteq K^{\perp}, A(G, U) \subseteq\left(\Lambda_{0}^{i} \times \prod_{j \neq i} \Omega_{p}^{j}\right) \cap G$ for all $i \in S$ except for finitely many indices.

In the rest of the proof we would like to show that $\widehat{G} \simeq \sum_{i \in S}\left(\Omega_{p}^{i}: \Delta_{p}^{i}\right)$, hence conclude that $G \simeq \sum_{i \in S}\left(\Omega_{p}^{i}: \Delta_{p}^{i}\right)$ by taking duals.

First of all by Lemma 3.1, $\Omega_{p}=\left\langle\left\{u_{i}: i=0,1,2, \ldots\right\}\right\rangle^{-}$. For any fixed $j \in S$ and $n=0,1,2, \ldots$ let $v_{n}=\left\{x_{i}\right\} \in H$, where $x_{j}=u_{n}$ (definition of $u_{n}$ was given right before Lemma 5.12) and $x_{i}=0$ for $i \neq j$ (an obvious abuse of notation).

Now for any $j \in S$, identify $\Omega_{p}$ with its topological isomorphic image $\Omega_{p}^{j} \times \prod_{i \neq j}\{0\}$ and for any $y=\left(y_{i}\right) \in \prod_{i \in S} \Omega_{p}^{i}$ define $\chi_{m}^{j}(y)=\chi_{m}\left(y_{j}\right)$. Using Lemma 5.12, it is easy to show that each $\chi_{m}^{j}$ is a continuous character of $H$ for any fixed $j \in S$ and $m \geq 0$, and $\left\{\chi_{m}^{i}: m=0,1,2, \ldots ; i \in S\right\}$ separates points of $H$. 
Let $L_{i}$ be the closed subgroup of $\widehat{H}$ generated by all $\chi_{m}^{i}(m=0,1,2, \ldots)$. Then by Lemma 3.1, $L_{i} \simeq \Omega_{p}$. Let $M_{i}$ be the closed subgroup of $\widehat{H}$ generated by $\chi_{0}^{i}$. Then by the proof of Lemma 3.1 we know that $M_{i}$ is compact open in $L_{i}$ and $M_{i} \simeq \Delta_{p}$ under the same topological isomorphism.

Claim 1. $\left\{L_{i}: i \in S\right\}$ are topologically independent, hence $\left\{M_{i}: i \in S\right\}$ are topologically independent.

Proof. It suffices to show that $\left\{L_{i}: i \in S\right\}$ are topologically independent. For this purpose we need to show that $L_{i} \cap\left\langle\left\{L_{j}: j \neq i\right\}\right\rangle^{-}=\{0\}$ for any $i \in S$. Let $K_{i}=\left\langle\left\{\chi_{m}^{j}: j \neq i, m \geq 0\right\}\right\rangle$. It is easy to see that $\bar{K}_{i}=\left\langle\left\{L_{j}: j \neq i\right\}\right\rangle^{-}$. Now for any $\chi \in L_{i}$ with $\chi \neq 0$, since $L_{i} \simeq \Omega_{p} \simeq \widehat{\Omega}_{p}$ and by the way $H$ is defined, there exists $g=\left(\ldots, 0, t_{i}, 0, \ldots\right) \in H$ such that $\chi(g) \neq 1$. Let $\delta=|\chi(g)-1|$ and consider the open neighborhood $U$ of $\chi$ given by $U=\{\varphi \in \widehat{H}:|\varphi(g)-\chi(g)|<\delta / 2\}$. Since for any $j \neq i, \chi_{m}^{j}(g)=1 \forall m \geq 0$, we must have $\varphi(g)=1 \forall \varphi \in K_{i}$. Hence $\varphi(g)=1 \forall \varphi \in \bar{K}_{i}$. Therefore $\bar{K}_{i} \cap U=\emptyset$. So $L_{i} \cap \bar{K}_{i}=\{0\}$ and the claim is proved.

Claim 2. $\left\langle\left\{M_{i}: i \in S\right\}\right\rangle^{-}=K^{\perp}$.

Proof. If $\left\langle\left\{M_{i}: i \in S\right\}\right\rangle^{-} \neq K^{\perp}$, then since $\left\langle\left\{M_{i}: i \in S\right\}\right\rangle^{-} \subseteq K^{\perp}$ there exists some $\varphi \in K^{\perp} \backslash\left\langle\left\{M_{i}: i \in S\right\}\right\rangle^{-}$and $\eta \in\left(K^{\perp}\right)^{\wedge}$ such that $\eta \in\left(\left\langle\left\{M_{i}: i \in S\right\}\right\rangle^{-}\right)^{\perp}$ and $\eta(\varphi) \neq 1$. Now extend $\eta$ to a continuous character on $\widehat{H}$ (which is possible by $[3,24.12]$ ), still denoted by $\eta$. Then $\eta \neq 1$ and so we may assume that $\eta=\left(\ldots, z_{i}, \ldots\right) \in H$ by Pontryagin duality. For any $i \in S$, write $\eta=\bar{z}_{i}+w_{i}$, where $\bar{z}_{i}=\left(\ldots, 0, z_{i}, 0, \ldots\right)$ and $w_{i}=\left(y_{i}\right)$ with $y_{j}=z_{j}$ when $j \neq i$ and $y_{i}=0$. Since $\eta$ annihilates $M_{i}$, we must have $\eta\left(\chi_{0}^{i}\right)=1$, i.e., $\chi_{0}^{i}(\eta)=1$, or $\chi_{0}^{i}\left(\bar{z}_{i}\right) \chi_{0}^{i}\left(w_{i}\right)=1$. But $\chi_{0}^{i}\left(w_{i}\right)=1$, so we have $\chi_{0}^{i}\left(\bar{z}_{i}\right)=1$. This implies that $z_{i} \in \Lambda_{0}^{i}$ for each $i \in S$. So $\eta=\left(z_{i}\right) \in \prod_{i \in S} \Lambda_{0}^{i}=K$. Consequently, $\eta(\varphi)=\varphi(\eta)=1$, a contradiction.

Claim 3. $K^{\perp}=\bigoplus_{i \in S} M_{i}$.

Proof. For any open and compact subgroup $U$ of $K^{\perp}$ we have $A(G, U)$ $\subseteq\left(\Lambda_{0}^{i} \times \prod_{j \neq i} \Omega_{p}^{j}\right) \cap G$ for almost all $i \in S$. So $U=A(\widehat{G}, A(G, U)) \supseteq$ $A\left(\widehat{G},\left(\Lambda_{0}^{i} \times \prod_{j \neq i} \Omega_{p}^{j}\right) \cap G\right)$ for almost all $i \in S$. But by the way $M_{i}$ is defined we know that $M_{i} \subseteq A\left(\widehat{G},\left(\Lambda_{0}^{i} \times \prod_{j \neq i} \Omega_{p}^{j}\right) \cap G\right)$ for all $i \in S$. Therefore $M_{i} \subseteq U$ for almost all $i \in S$. So by Claims 1, 2 and Theorem 2.5 we know that $K^{\perp}=\bigoplus_{i \in S} M_{i}$.

Now by applying Proposition 5.5 to $\left\{L_{i}: i \in S\right\}$ and $\left\{M_{i}: i \in S\right\}$ we conclude that the local direct product $\sum_{i \in S}\left(L_{i}: M_{i}\right)$ is topologically isomorphic to an open (hence closed) subgroup $L$ of $\widehat{G}$ generated by all $L_{i}$ 's 
and $K^{\perp}$. Since $L$ separates points of $G$, we must have $L=\widehat{G}$. Therefore $\widehat{G} \simeq \sum_{i \in S}\left(L_{i}: M_{i}\right) \simeq \sum_{i \in S}\left(\Omega_{p}^{i}: \Delta_{p}^{i}\right)$. So by taking duals we know that $G \simeq \sum_{i \in S}\left(\Omega_{p}^{i}: \Delta_{p}^{i}\right)$.

Now we can display our example that shows topological independence (TI) does not imply strong topological independence (STI).

ExAmple 3. As in Example 1 let $H=\left\{x \in \prod_{i=1}^{\infty} \Omega_{p}^{i}: \exists m \in N\right.$ such that $p^{m} x \in \prod_{i=1}^{\infty} \Delta_{p}^{i}$, where $\Omega_{p}^{i}=\Omega_{p}$ and $\Delta_{p}^{i}=\Delta_{p} \forall i \geq 1$; then topologize $H$ so that $K=\prod_{i=1}^{\infty} \Delta_{p}^{i}$ is an open compact subgroup of $H$.

Now we can construct a family $\left\{M_{i}\right\}_{i \in S}$ of subgroups of $\widehat{H}$ as in the proof of the necessity part of the previous theorem and let our group $G$ be the closure of the subgroup generated by all $M_{i}$ 's, where $|S|=\aleph_{0}$. Then for this $G$ and all these $M_{i}$ 's TI does not imply STI. Indeed, by Claim 2 we know that $G$ is compact. On the other hand, it is not difficult to show that $H / K$ is uncountable, hence $G=K^{\perp}=(H / K)^{\wedge} \simeq \prod_{\alpha \in A} \Delta_{p}^{\alpha}$ with $A$ being uncountable. Therefore TI does not imply STI (otherwise by Theorem 2.4 $G \simeq \prod_{i \in S} M_{i} \simeq \prod_{i \in S} \Delta_{p}^{i}$, which is impossible since $\left.|A|>|S|\right)$.

Now we can state a generalization of Theorem 5.9; its proof is similar to that of Theorem 5.9.

THEOREM 5.14. Let $G$ be a torsion-free LCA group. Then $G$ is self-dual and has the finite property if and only if

$$
G \simeq R^{n} \times D \times \widehat{D} \times \sum_{p \in \wp}\left(\sum_{i \in S_{p}}\left(\Omega_{p}^{i}: \Delta_{p}^{i}\right): \Delta_{p}^{\mu_{p}}\right)
$$

where $n$ is a non-negative integer; $D$ is a divisible torsion-free discrete abelian group; $\wp$ is a set of prime numbers and $S_{p}$ is an arbitrary index set with cardinality $\mu_{p}$ for each $p \in \wp ; \Omega_{p}^{i}=\Omega_{p}$ and $\Delta_{p}^{i}=\Delta_{p} \forall i \in S_{p}$.

6. Self-dual divisible LCA groups. Although we can prove directly that any self-dual divisible topological $p$-group is a finite product $\Omega_{p}^{\mu}$ of the group $\Omega_{p}$ of $p$-adic numbers and then derive the structure of self-dual divisible LCA groups by employing Lemmas 10 and 11 of [5], we shall accomplish our goal by an indirect approach. By considering torsion-free divisible LCA groups instead of self-dual divisible ones (self-duality and divisibility of an LCA group force it to be torsion-free) we discovered that any divisible torsion-free topological $p$-group is actually the subgroup $B_{p}(\mu)$ of $\Omega_{p}^{\mu}$, for some cardinal number $\mu$, that consists of all bounded elements endowed with an appropriate topology. This not only gives us an easier way of proving some properties of the minimal divisible extension of a torsion-free topological $p$-group, but also exhibits the structure of divisible torsion-free LCA groups (see Theorem 6.9) and bi-divisible LCA groups (see Theorem 6.13) 
so that the structure theorem of self-dual divisible LCA groups follows immediately as a corollary.

LEMMA 6.1. Let $G=\sum_{i \in S}\left(G_{p_{i}}: H_{p_{i}}\right)$, where $p_{i}$ 's are distinct primes and each $G_{p_{i}}$ is a divisible topological $p_{i}$-group. Then $G$ is divisible.

\section{P r o of. Straightforward.}

Let $B_{p}(\mu)=\left\{\left(x_{i}\right) \in \prod_{i \in S} \Omega_{p}^{i}: \exists k \geq 0\right.$ such that $\left.p^{k} x_{i} \in \Delta_{p} \forall i \in S\right\}$, where $\mu=|S|$; then topologize $B_{p}(\mu)$ so that $\prod_{i \in S} \Delta_{p}^{i}$ is an open and compact subgroup of $B_{p}(\mu)$.

Remark. (1) $B_{p}(\mu)$ actually consists of all bounded elements of $\prod_{i \in S} \Omega_{p}^{i}$, i.e., $B_{p}(\mu)=\bigcup_{k=0}^{\infty} \prod_{i \in S} \Lambda_{-k}^{i}$ as a set.

(2) $B_{p}(\mu)$ itself is obviously a divisible torsion-free topological $p$-group. In the following we are going to show that any divisible torsion-free topological $p$-group is of this form. Therefore we can be pleased that we understand divisible torsion-free topological $p$-groups completely.

(3) $B_{p}(\mu)$ is a generalization of a finite direct product of copies of $\Omega_{p}$, i.e., when $|S|<\aleph_{0}, B_{p}(\mu)=\prod_{i=1}^{|S|} \Omega_{p}^{i}$. When $|S| \geq \aleph_{0}, B_{p}(\mu)$ contains the local direct product $\sum_{i \in S}\left(\Omega_{p}^{i}: \Delta_{p}^{i}\right)$ as an open subgroup.

TheOREM 6.2. Let $G$ be an $L C A$ group. Then $G$ is a divisible torsion-free topological p-group if and only if $G \simeq B_{p}(\mu)$ for some cardinal number $\mu$.

Proof. The sufficiency is clear. To show the necessity, note that, by Proposition 5.6, $G$ can be regarded as an algebraic subgroup of $\prod_{i \in S} \Omega_{p}^{i}$ for some index set $S$, and $G$ has an open and compact subgroup $K$ that is topologically isomorphic to $\prod_{i \in S} \Delta_{p}^{i}$. Let $\tau$ be the algebraic isomorphism of $G$ into $\prod_{i \in S} \Omega_{p}^{i}$ with $\left.\tau\right|_{K}: K \simeq \prod_{i \in S} \Delta_{p}^{i}$. It suffices to show that $\tau(G)=B_{p}(\mu)$, which is pretty straightforward.

By applying Theorem 6.2 we obtain a concrete description of the minimal divisible extension of a torsion-free topological $p$-group, especially that of $\Delta_{p}^{\mu}$.

COROllary 6.3. Let $E$ be the minimal divisible extension of a torsionfree topological p-group $G$. Then $E$ is topologically isomorphic to $B_{p}(\mu)$ for some cardinal number $\mu$.

Proof. Since $E$ is the minimal divisible extension of a torsion-free topological $p$-group, it is also a torsion-free topological $p$-group by $[1,2.15]$. So by Theorem 6.2 we know that $E$ is topologically isomorphic to a $B_{p}(\mu)$ for some cardinal number $\mu$.

Remark. When $G$ in Theorem 6.3 is compact it is well known that $G \simeq \Delta_{p}^{\mu}$. Therefore $B_{p}(\mu)$ is exactly the minimal divisible extension of $\Delta_{p}^{\mu}$ studied by Robertson in [7]. 
COROLLARY 6.4. Any torsion-free topological p-group can be regarded as an open subgroup of $B_{p}(\mu)$ for some cardinal $\mu$.

Proof. Let $E$ be the minimal divisible extension of $G$. Then $E$ is topologically isomorphic to $B_{p}(\mu)$ for some cardinal $\mu$ by Corollary 6.3. But $G$ is an open subgroup of $E$ (see [1, p. 31]); therefore $G$ can be regarded as an open subgroup of $B_{p}(\mu)$.

Since any torsion-free topological $p$-group $G$ contains an open and compact subgroup $K \simeq \Delta_{p}^{\mu}$ and $G$ can be regarded as an open subgroup of $B_{p}(\mu)$, which is the minimal divisible extension of $\Delta_{p}^{\mu}$, one can see easily that $B_{p}(\mu)$ is also the minimal divisible extension of $G$. Actually, we shall prove that $B_{p}(\mu)$ is the minimal divisible extension of any of its open subgroups (this was also proved by Robertson in [7] in a slightly different way).

Corollary 6.5. $B_{p}(\mu)$ is the minimal divisible extension of any of its open subgroups.

Proof. Let $H$ be any open subgroup of $B_{p}(\mu)$; then $B_{p}(\mu) / H$ is a discrete divisible torsion group since $B_{p}(\mu)$ is a divisible topological $p$-group. Therefore by [3, A.17], $B_{p}(\mu)$ is the minimal divisible extension of $H$.

Also, in [7] Robertson showed that the character group of the minimal divisible extension of $\Delta_{p}^{\mu}$ is divisible if and only if $\mu<\aleph_{0}$. Since we now know the structure of the minimal divisible extension of $\Delta_{p}^{\mu}$, we can give a simpler proof of the result.

Lemma 6.6. If $\mu \geq \aleph_{0}$, then $B_{p}(\mu) / \Delta_{p}^{\mu} \simeq \sum_{i \in A} Z^{i}\left(p^{\infty}\right)$ with $|A|=2^{\mu}$.

Proof. Since $B_{p}(\mu) / \Delta_{p}^{\mu}$ is a divisible discrete topological $p$-group, $B_{p}(\mu) / \Delta_{p}^{\mu} \simeq \sum_{i \in A} Z^{i}\left(p^{\infty}\right)$. But $\Lambda_{-1}^{\mu} \subseteq B_{p}(\mu)$ (here $\Lambda_{-1}^{\mu}=\prod_{i \in S} \Lambda_{-1}^{i}$ and $|S|=\mu)$ and $\left|\Lambda_{-1}^{\mu} / \Delta_{p}^{\mu}\right|=2^{\mu}$. Hence $|A|=2^{\mu}$.

Here is Robertson's result.

Proposition 6.7. $\widehat{B}_{p}(\mu)$ is divisible if and only if $\mu<\aleph_{0}$.

Proof. The sufficiency is obvious. For necessity, suppose that $\widehat{B}_{p}(\mu)$ is divisible, and $\mu \geq \aleph_{0}$. Let $K=\Delta_{p}^{\mu}$. Then by Lemma $6.6, B_{p}(\mu) / K \simeq$ $\sum_{i \in A} Z^{i}\left(p^{\infty}\right)$ with $|A|=2^{\mu}$. Now $K^{\perp}=\left(B_{p}(\mu) / K\right)^{\wedge} \simeq \prod_{i \in A} \Delta_{p}^{i}$. Since $\widehat{B}_{p}(\mu)$ is also a topological $p$-group by $[3,2.15]$, we have $\widehat{B}_{p}(\mu)=B_{p}(\lambda)$ by Corollary 6.3 and Corollary 6.5 with $\lambda=|A|$. But again by Lemma 6.6 $B_{p}(\lambda) / K^{\perp}=\sum_{i \in B} Z^{i}\left(p^{\infty}\right)$ with $|B|=2^{\lambda}>\mu$. On the other hand, by taking duals we have $K \simeq\left(B_{p}(\lambda) / K^{\perp}\right)^{\wedge} \simeq \prod_{i \in B} \Delta_{p}^{i}$. So $\Delta_{p}^{\mu} \simeq \Delta_{p}^{|B|}$; hence $\mu=|B|$ by Lemma 3.2 , which is impossible since we already know that $|B|>\mu$ from the above discussion. Therefore $\mu<\aleph_{0}$. 
Lemma 6.8. Let $G$ be an LCA group. Then $G$ is a divisible torsion-free topological torsion group if and only if $G \simeq \sum_{p \in \wp}\left(B_{p}\left(\mu_{p}\right): \Delta_{p}^{\mu_{p}}\right)$, where $\wp$ is a set of prime numbers and $\mu_{p}$ is a cardinal number for each $p \in \wp$.

Proof. The sufficiency follows from Lemma 6.1 immediately. To show the necessity, let $H$ be an open and compact subgroup of $G$ (which is possible by $[1,3.5]$ since $G$ is totally disconnected). Then by [1, 3.13], $G \simeq \sum_{p \in \wp}\left(G_{p}: H_{p}\right)$, where $G_{p}$ is the $p$-component of $G$ and $H_{p}=H \cap G_{p}$. Since $G$ is divisible and each $G_{p}$ is a direct summand in the decomposition, $G_{p}$ must be divisible. Therefore by Theorem $6.2, G_{p} \simeq B_{p}\left(\mu_{p}\right)$ with $H_{p} \simeq \Delta_{p}^{\mu_{p}}$ under the same isomorphism.

Now we are ready to prove the structure theorem of divisible torsion-free LCA groups.

TheOrem 6.9. Let $G$ be an LCA group. Then $G$ is divisible torsion-free if and only if

$$
G \simeq R^{n} \times Q^{a *} \times \widehat{Q}^{b} \times \sum_{p \in \wp}\left(B_{p}\left(\mu_{p}\right): \Delta_{p}^{\mu_{p}}\right),
$$

where $n$ is a non-negative integer; $a$ and $b$ are cardinal numbers; $\wp$ is a set of prime numbers and $\mu_{p}$ is a cardinal number for each $p \in \wp$.

Pr o of. The sufficiency is clear since each direct summand is divisible and torsion-free. Towards the necessity, note that by [1, Proposition 9.5], $G \simeq R^{n} \times Q^{a *} \times \widehat{Q}^{b} \times E$, where $E$ is a topological torsion group. Being a direct summand, $E$ must be divisible since $G$ is divisible. Therefore by Lemma 6.8 we have $E \simeq \sum_{p \in \wp}\left(B_{p}\left(\mu_{p}\right): \Delta_{p}^{\mu_{p}}\right)$.

Now by using Theorem 6.2 we can show that the divisibility of a topological $p$-group $G$ and its character group $\widehat{G}$ together imply the self-duality of $G$ automatically (see Lemma 6.11). As a consequence, for any torsionfree topological torsion group the self-duality and divisibility of $G$ turn out to be equivalent to the divisibility of $G$ and its character group $\widehat{G}$ (see Lemma 6.12). Because of this, the structure of a bi-divisible LCA group can be described explicitly.

Definition 6.10. Let $G$ be an LCA group. Then $G$ is said to be $b i$ divisible if $G$ and its character group $\widehat{G}$ are both divisible.

Lemma 6.11. Let $G$ be a topological p-group. Then the following are equivalent:

(1) $G \simeq \Omega_{p}^{m}$, where $m$ is a non-negative integer;

(2) $G$ is self-dual divisible;

(3) $G$ is bi-divisible; 
(4) $G$ is self-dual and is (topologically isomorphic to) an open subgroup of $\Omega_{p}^{m}$ for some non-negative integer $m$.

Proof. $(1) \Rightarrow(2) \Rightarrow(3)$ is clear. $\quad(3) \Rightarrow(1)$ follows from Theorem 6.2 and Proposition 6.7. Since $(1) \Rightarrow(4)$ is immediate, it remains to show that $(4) \Rightarrow(1)$. Note that being an open subgroup of $\Omega_{p}^{m}, G$ must be metrizable. Therefore by Lemma $4.2, G \simeq \sum_{i \in S}\left(\Omega_{p}^{i}: \Delta_{p}^{i}\right)$, where $S$ is countable. So the open compact subgroup $\Delta_{p}^{\mu}$ (where $\left.\mu=|S|\right)$ of $\sum_{i \in S}\left(\Omega_{p}^{i}: \Delta_{p}^{i}\right)$ is topologically isomorphic to an open and compact subgroup $K$ of $\Omega_{p}^{m}$ that is topologically isomorphic to $\Delta_{p}^{m}$ by $[1,2.23]$. Therefore $\Delta_{p}^{\mu} \simeq \Delta_{p}^{m}$. So by Lemma 3.2 we must have $\mu=m$ and the proof is finished.

LEMMA 6.12. Let $G$ be a topological torsion group. Then the following are equivalent:

(1) $G \simeq \sum_{p \in \wp}\left(\Omega_{p}^{m_{p}}: \Delta_{p}^{m_{p}}\right)$, where $\wp$ is a set of prime numbers and each $m_{p}$ is a non-negative integer for $p \in \wp$;

(2) $G$ is self-dual and divisible;

(3) $G$ is bi-divisible.

Proof. $(1) \Rightarrow(2) \Rightarrow(3)$ is clear. For $(3) \Rightarrow(1)$, note that by Lemma 6.8 we have $G \simeq \sum_{p \in \wp}\left(B_{p}\left(\mu_{p}\right): \Delta_{p}^{\mu_{p}}\right)$. Then by taking duals we have

$$
\widehat{G} \simeq \sum_{p \in \wp}\left(\widehat{B}_{p}\left(\mu_{p}\right): A\left(\widehat{B}_{p}\left(\mu_{p}\right), \Delta_{p}^{\mu_{p}}\right)\right) .
$$

Since $\widehat{G}$ is assumed to be divisible, each $\widehat{B}_{p}\left(\mu_{p}\right)$ must also be divisible. So both $B_{p}\left(\mu_{p}\right)$ and $\widehat{B}_{p}\left(\mu_{p}\right)$ are divisible. Hence by Lemma 6.11 we have $B_{p}\left(\mu_{p}\right) \simeq \Omega_{p}^{m_{p}}$ for some non-negative integer $m_{p}$. So (3) implies (1) and the lemma is proved.

TheOREM 6.13. Let $G$ be an LCA group. Then $G$ is bi-divisible if and only if

$$
G \simeq R^{n} \times Q^{a *} \times \widehat{Q}^{b} \times \sum_{p \in \wp}\left(\Omega_{p}^{m_{p}}: \Delta_{p}^{m_{p}}\right),
$$

where $n$ is a non-negative integer; $a$ and $b$ are cardinals; $\wp$ is a set of prime numbers and for each $p \in \wp, m_{p}$ is a non-negative integer.

Proof. $\Leftarrow$ is clear. For $\Rightarrow$, note that the divisibility of $\widehat{G}$ implies that $G$ is torsion-free by $[2,24.23]$. Therefore by [1, Proposition 9.5] we know that

$$
G \simeq R^{n} \times Q^{a *} \times \widehat{Q}^{b} \times E,
$$

where $n$ is a non-negative integer; $a$ and $b$ are cardinals; and $E$ is a densely divisible torsion-free topological torsion group. Since $G$ is divisible, the direct summand $E$ must be divisible. Also by taking duals we have

$$
\widehat{G} \simeq R^{n} \times Q^{b *} \times \widehat{Q}^{a} \times \widehat{E} .
$$


Since $\widehat{G}$ is assumed to be divisible, the direct summand $\widehat{E}$ must be divisible. Therefore $E$ and $\widehat{E}$ are both divisible. Since $E$ is a topological torsion group, by Lemma 6.12 we know that $E \simeq \sum_{p \in \wp}\left(\Omega_{p}^{m_{p}}: \Delta_{p}^{m_{p}}\right)$, where $\wp$ is a set of prime numbers and each $m_{p}$ is a non-negative integer for $p \in \wp$. Hence

$$
G \simeq R^{n} \times Q^{a *} \times \widehat{Q}^{b} \times \sum_{p \in \wp}\left(\Omega_{p}^{m_{p}}: \Delta_{p}^{m_{p}}\right)
$$

Corollary 6.14. Let $G$ be an LCA group. Then $G$ is self-dual divisible if and only if $G \simeq R^{n} \times Q^{a *} \times \widehat{Q}^{a} \times \sum_{p \in \wp}\left(\Omega_{p}^{m_{p}}: \Delta_{p}^{m_{p}}\right)$, where $n$ is a nonnegative integer; $a$ is a cardinal; $\wp$ is a set of prime numbers and for each $p \in \wp, m_{p}$ is a non-negative integer.

Proof. It suffices to show that $\Rightarrow$ holds. Suppose that $G$ is self-dual divisible. Then $G$ is of course bi-divisible. Hence by Theorem 6.13

$$
G \simeq R^{n} \times Q^{a *} \times \widehat{Q}^{b} \times \sum_{p \in \wp}\left(\Omega_{p}^{m_{p}}: \Delta_{p}^{m_{p}}\right) .
$$

Now by taking duals we have

$$
\widehat{G} \simeq R^{n} \times \widehat{Q}^{a} \times Q^{b *} \times \sum_{p \in \wp}\left(\Omega_{p}^{m_{p}}: \Lambda_{-1}^{m_{p}}\right) .
$$

Since isomorphic topological groups have isomorphic identity components, we must have $R^{n} \times \widehat{Q}^{b} \simeq R^{n} \times \widehat{Q}^{a}$. Again by taking duals we obtain that $R^{n} \times Q^{b *} \simeq R^{n} \times Q^{a *}$. This implies that $a=b$ and the corollary is proved.

Note. I would like to thank Dr. M. A. Khan who kindly referred me to his paper $A$ theorem on power-open LCA groups and its consequences, Bull. Austral. Math. Soc. 26 (1982), 239-247, in which this corollary was obtained by another method.

Because of Corollary 6.14 we can derive some interesting results about group extensions.

COROLlary 6.15. Let $G$ be an LCA group that is a divisible self-dual extension of $\prod_{i=1}^{n} \Delta_{p}^{i}$ by $\sum_{i=1}^{n} Z\left(p^{\infty}\right)$. Then $G \simeq \prod_{i=1}^{n} \Omega_{p}^{i}$.

COROLlary 6.16. Let $G$ be an LCA group that is a self-dual extension of $\prod_{i \in S} \Delta_{p}^{i}$ by $\sum_{i \in S} Z\left(p^{\infty}\right)$. Then $G$ is not divisible if $|S| \geq \aleph_{0}$.

From Corollary 6.14 we can get the structure of self-dual divisible connected groups without any difficulty.

COROLlary 6.17. Let $G$ be a self-dual divisible LCA group. Then $G$ is connected if and only if $G \simeq R^{n}$, where $n$ is a non-negative integer.

Even though we have not been able to classify all self-dual torsion-free LCA groups, we shall be content temporarily with the following findings. 
Proposition 6.18. Any torsion-free densely divisible, especially any selfdual torsion-free, $L C A$ group $G$ can be regarded as an open subgroup of an $L C A$ group of the form $R^{n} \times Q^{a *} \times \widehat{Q}^{b} \times \sum_{p \in \wp}\left(B_{p}\left(\mu_{p}\right): \Delta_{p}^{\mu_{p}}\right)$, where $n$ is a non-negative integer; $a$ and $b$ are cardinal numbers; $\wp$ is a set of prime numbers and $\mu_{p}$ is a cardinal number for $p \in \wp$.

Proof. Let $G$ be torsion-free and densely divisible; then by $[1,3.13]$ and $[1,9.5]$

$$
G \simeq R^{n} \times Q^{a *} \times \widehat{Q}^{b} \times \sum_{p \in \wp}\left(H_{p}: K_{p}\right),
$$

where $n$ is a non-negative integer; $a$ and $b$ are cardinals; $\wp$ is a set of prime numbers and each $H_{p}$ is a torsion-free topological $p$-group with the compact open subgroup $K_{p} \simeq \Delta_{p}^{\mu_{p}}$ for some cardinal $\mu_{p}$. It is straightforward to show that $R^{n} \times Q^{a *} \times \widehat{Q}^{b} \times \sum_{p \in \wp}\left(B_{p}\left(\mu_{p}\right): \Delta_{p}^{\mu_{p}}\right)$ is the minimal divisible extension of $G$. So the first statement of the proposition is proved. The second statement follows from the first statement and the fact that selfduality and torsion-freeness imply the dense divisibility.

\section{References}

[1] D. L. Armacost, The Structure of Locally Compact Abelian Groups, Marcel Dekker, New York 1981.

[2] L. Corwin, Some remarks on self-dual locally compact Abelian groups, Trans. Amer. Math. Soc. 148 (1970), 613-622.

[3] E. Hewitt and K. A. Ross, Abstract Harmonic Analysis I, Springer, New York 1979.

[4] L. S. Pontryagin, Topological Groups, 2nd ed., Gordon and Breach, New York 1966.

[5] M. Rajagopalan and T. Soundararajan, Structure of self-dual torsion-free metric LCA groups, Fund. Math. 65 (1969), 309-316.

[6] N. W. Rickert, The structure of a class of locally compact abelian groups, unpublished (1968).

[7] L. C. Robertson, Connectivity, divisibility, and torsion, Trans. Amer. Math. Soc. 128 (1967), 482-505.

[8] N. Ya. Vilenkin, Direct decomposition of topological groups, Amer. Math. Soc. Transl. (1) 8 (1962), 79-185.

DEPARTMENT OF MATHEMATICS

UNIVERSITY OF OREGON

EUGENE, OREGON 97403

U.S.A. 